\title{
Hepatocellular Carcinoma: Review of Current Treatment with a Focus on Transarterial Chemoembolization and Radiofrequency Ablation
}

\author{
Nikhar Chhaniwal' ${ }^{1}$, Cheng Li'1 , Jiaxiang Wang1, Guosheng Qiang1, Tingyue $\mathbf{i}^{2}$, Hendi Maher ${ }^{3}$ \\ ${ }^{1}$ Department of Interventional Radiology, Yangzhou Peoples No.1 Hospital Affiliated to Yangzhou University, \\ Yangzhou, China \\ ${ }^{2}$ Department of Ultrasound, Yangzhou Peoples No.1 Hospital Affiliated to Yangzhou University, Yangzhou, \\ China \\ ${ }^{3}$ Department of General Surgery, Sir Run Run Shaw Hospital Hangzhou, College of Medicine, \\ Zhejiang University, Hangzhou, China \\ Email: Nikharujjain@yahoo.com
}

Received 23 January 2015; accepted 24 March 2015; published 26 March 2015

Copyright @ 2015 by authors and Scientific Research Publishing Inc.

This work is licensed under the Creative Commons Attribution International License (CC BY).

http://creativecommons.org/licenses/by/4.0/

(c) (i) Open Access

\section{Abstract}

Hepatocellular carcinoma (HCC) is one of the most deadly tumors and its incidence has been rising worldwide. In its various stages, several modalities of treatment and therapies have been developed. Appropriate HCC treatment needs to consider the tumor stage, underlying liver disease and patient's performance levels. Liver transplantation is an effective treatment but unreliable due to scarcity of donors, whereas surgical resection possesses the potential to achieve long-term survival. However, most tumors are not resectable due to their size, location, number and the function of the liver being compromised. Thus, resection is the first choice treatment for resectable tumors only. This prompts the rise of transarterial chemoembolization (TACE) for unresectable HCC. Progressively, radiofrequency ablation (RFA) arose though it exhibits high postoperative recurrence and cancer mortality. Notably, identifying the best-suited treatment is critical in enabling maximum long-term survival. Though HCC is an identified chemoresistant tumor, chemotherapies have been developed for advanced HCC. Among them, sorafenib, blocks tumor cell proliferation thus improving patient survival, even though costly. In comparison to RFA, TACE has been identified to have more complications and symptoms. However, it demonstrates improved survival benefits and quality of patient care more than symptomatic supportive care. Furthermore, for unresectable HCC, TACE can replace the costly and modestly effective sorafenib treatment; whereas RFA is potentially curative in treating HCC and an alternative to liver transplantation or surgical resection in the early HCC stages. It treats primary and secondary liver tumor in the very early 
stage HCC. Its reliance on image guidance is minimally invasive and enhances its efficacy and safety, especially where surgery will not have been possible. However, skin burns, hemorrhage, hepatic abscess and pleural effusion are complications that accompany RFA prompting the need for additional treatment.

\title{
Keywords
}

\author{
Hepatocellular Carcinoma, Tumor, Treatment, Transarterial Chemoembolization, Radiofrequency \\ Ablation
}

\section{Introduction}

Hepatocellular carcinoma (HCC) is a major cause of cancer death [1]. It is the most frequent primary malignancy of the liver while it has close links to chronic liver disease [2]. It is the sixth leading cancer globally and the third highest cause of cancer-linked deaths. HCC is a complex condition with numerous risk factors associated with it, which makes it difficult to evaluate and treat through a number of treatment and therapies which have been developed [3]. Patients who are considered to be at high risk of having this disease include those with cirrhosis or underlying chronic liver disease, with the disease evolving through a multistep development of hepatocarcinogenesis that ranges from regenerative nodules to becoming hypervascular HCCs. It has been demonstrated that there is a likelihood of borderline hepatocellular nodules including high-grade dysplastic nodules (DN) or early HCC to be considered as precursors of progressed HCCs [4].

The Barcelona Clinic Liver Cancer (BCLC) staging system is the most commonly used staging system and has been endorsed by the most liver associations. In the BCLC classification, HCC patients are categorized into five stages that include $0, \mathrm{~A}, \mathrm{~B}, \mathrm{C}$ and D based on pre-established prognostic variables and it allocates therapies based on treatment-related status. Thus, the staging system provides information not only about prognostic prediction, but also regarding treatment allocation. Notably, prognosis prediction is understood based on valuables that are linked to the status of the tumor (such as number, size, metastasis, vascular invasion), health status and liver function. Whereas, treatment allocation involves the integration of treatment dependant variables that have demonstrated the ability of influencing therapeutic outcome such as Portal Hypertension, Bilirubin or presence of symptoms [5] (in Table 1 [6]).

Surgical resection has conventionally been proven to have the highest potential of enabling long-term survival. However, with more than $70 \%$ of tumors in HCC patients not resectable because of the location, size, number and the function of the liver being compromised. TACE was once widely acknowledged as an alternative treatment for unresectable HCC. Lately, however, local mesenchymal treatment, such as the RFA, have been developed and proceeded to gain considerable recognition and are widely applied [7]. Courtesy of the high postoperative recurrence and cancer mortality, the prognosis of HCC patients has previously proven discouraging. Therefore, the identification of the optimal therapy for HCC is crucial in facilitating maximum long-term survival [8].

\section{Incidence}

It is estimated that 696,000 deaths in 2008 were because of HCC, with $85 \%$ of HCC cases occurring in developing countries [3]. In particular, high incidence rates of HCC are identified in sub-Saharan Africa, China and Southeast Asia. Globally, the HCC incidence is evidently increasing [9]. In the USA and Europe, the incidence rates are lower but have been on the rise over the recent decades [10]. The median age for HCC diagnosis was identified to stand at 64 years in the 2001-2006 period. However, the age distribution has been identified to shift downward with the greatest proportional increase in HCC incidence identified to be among individuals aged 45 60 years. At the same time, however, incidence remains highest and on the increase among older patients [3]. Worth noting, hepatitis B and C are identified to be the main worldwide causes of HCC. However, it is also believed that alcohol-linked cirrhosis and NASH cirrhosis also contribute [11].

\section{Treatment}

To begin with, surveillance programs of those at risk have been developed in numerous regions of the world. 
Table 1. Classification of the BCLC staging for HCC.

\begin{tabular}{|c|c|c|c|c|}
\hline \multicolumn{5}{|c|}{ BCLC staging for HCC } \\
\hline \multirow{2}{*}{ Stage } & \multirow{2}{*}{ Pst } & Tumor status & & \multirow{2}{*}{ Liver function status } \\
\hline & & Tumor stace & Oluuda stago & \\
\hline Stage A: early HCC & 0 & & & \\
\hline A1 & 0 & Single, $<5 \mathrm{~cm}$ & I & $\begin{array}{c}\text { No portal hypertension and normal } \\
\text { bilirubin }\end{array}$ \\
\hline A2 & 0 & Single, $<5 \mathrm{~cm}$ & I & $\begin{array}{l}\text { portal hypertension and normal } \\
\text { bilirubin }\end{array}$ \\
\hline A3 & 0 & Single, $<5 \mathrm{~cm}$ & I & $\begin{array}{l}\text { portal hypertension and abnormal } \\
\text { bilirubin }\end{array}$ \\
\hline A4 & 0 & 3 tumours $<3 \mathrm{~cm}$ & I & Child-pugh A-B \\
\hline Stage B: intermediate stage & 0 & Large multinodular & I-II & Child-pugh A-B \\
\hline Stage C: advanced stage & $1-2^{*}$ & $\begin{array}{l}\text { Vascular invasion or extra } \\
\text { hepatic spread }^{\mathrm{a}}\end{array}$ & I-II & Child-pugh A-B \\
\hline Stage D: end-stage HCC & $3-4^{b}$ & Any & $\mathrm{III}^{\mathrm{b}}$ & Child-pugh $\mathrm{C}^{\mathrm{b}}$ \\
\hline
\end{tabular}

Stage A and B: all criteria ought to be fulfilled. Stage C: the least being one criterion PST 1-2 or extrahepatic spread/vascular invasion. Stage D: the least being one criterion; PST 3-4.

Consequently, this has made it possible to diagnose an increased proportion of HCCs at early stages. In these cases, the best theoretical treatment would be liver transplantation, but this treatment is limited because of the scarcity of donors. Currently, RFA and hepatic resection (HR) are considered to be the first line therapies that are potentially curative in treating HCC and are preferable in the cases where transplantation is not applicable as an option for the patients on the waiting list [2]. The RFA or surgical resection are considered for patients who are in the early phase of HCC, while TACE would be the first-line treatment applying among patients with intermediate HCC [12]. Nonetheless, recurrence of HCC is observed to be common, exhibiting cumulative 5-year recurrence exceeding $70 \%$ even when the tumor ablation by RFA is effective. Thus, it is critical to first identify those patients at a high risk of tumor recurrence after being treated. This prompts the development of recurrence predictive models to facilitate the following up of high-risk patients with more frequency that exceed routine schedule. The models would be critical in supporting clinical decision-making in following up the patients [13].

The concern that arises in the treatment of HCC is that curative treatments are not feasible as patients present with intermediate or advances stages of the disease [14]. However, in all cases, the appropriate management need to take into account the tumor stage, patient's fitness levels and the degree of underlying chronic liver disease because of their independent prognostic significance and potential of negatively affecting liver parenchyma in a manner that make adequate hepatic deserve to be a requisite or safe systemic and loco regional therapy [10].

\section{Surgical Resection}

Surgical resection is the 'gold standard' treatment for HCC patients. When technically feasible, it provides a real chance of long-term cure or survival [15]. Over the recent decades, its safety has been improved leading to reduction of mortality rate following hepatic resection to be less that than $5 \%$ in experienced centers. These have extended even for advanced HCCs, with complicated hepatic resection made safely possible due to advances achieved in surgical techniques. Several criteria are used in determining the consideration of surgical resection and these are based on the tumor status and liver function. The method that is adopted in conducting the preoperative evaluation of the liver function that determines the extent the resection is likely to vary among institutions and districts [16].

However, in numerous patients, resection is not appropriate or possible as a result due to liver dysfunction caused by cirrhosis, technical difficulties or concomitant medical problems [15]. Furthermore, even though suitable for HCC patients with cirrhosis, it is unsuitable for HCC patients having clinically significant portal hypertension (PHT) due to the potential for postoperative hepatic decompensation. While PHT occurs in 25\% - 
$55 \%$ of HCC and cirrhosis, the absence of PHT is considered the best predictor of excellent hepatic resection outcomes. In their guidelines, most American and European Associations do not recommend hepatic resection as an option for patients having PHT [17].

\section{Liver Transplantation}

Liver transplantation is the most attractive therapeutic option in treating HCC, as it can remove the detectable as well as the undetectable tumor nodules and those pre-neoplastic lesions located in the cirrhotic liver. Furthermore, liver transplantation treats the underlying cirrhosis while preventing the distant or postoperative complications linked to liver failure and portal hypertension from developing [18]. The Milan criteria have served as the most accepted criteria for determining the HCC patients that are potential candidates of undergoing liver transplantation. Numerous studies have validated the criteria with those patients that meet the Milan criteria observed to exhibit significantly higher rates of survival than those that do not meet the criteria, with the comparison for 3-year survival rate being between $79 \%$ - 91\% and 60\% - 66\%. For living donor liver transplantation, the selection criteria can be expanded more readily considering the strong motivation of giving and dedication to recipients that donors are expected to have [16] (in Table 2 [19]).

However, using the liver transplantation alone has been found to give disappointing results, as it has recorded a 1-year recurrence rate of $30 \%-60 \%$, with the 5 -year survival rate recorded to be $<30 \%$ [18].

\section{Chemotherapeutic Agents}

HCC has been identified as a chemo-resistant tumor. The multidrug resistance protein expression and drug efflux mechanisms have been identified to render the chemotherapeutic agents only minimally effective. This has prompted the need to combine chemotherapy regimens and adoption of targeted therapy [11]. Consequently, systemic chemotherapies are provided to most patients having advanced HCC. Doxorubicin was in the past considered routinely for advanced HCC but was found ineffective as it response rate was around $15 \%-20 \%$. The rest of the chemotherapy agents such as cisplatin, epirubicin, etoposide, 5-fluorouracil and their combinations exhibit lower efficacy [20].

Sorafenib, an orally active multikinase inhibitor, has been identified to block both tumor cell proliferations. In preclinical studies, Sorafenib has exhibited antiproliferation activity in vitro, while significantly inhibiting tumor growth as well as angiogenesis and inducing tumor cells apoptosis [10]. It is the first agent that is exhibited to improve overall survival in advanced HCC. It achieves this by inhibiting angiogenesis by targeting receptors for vascular endothelial growth factor (VEGF) receptor (VEGFR2 and VEGFR3) and blocking tumor cell proliferation through the inhibition of RAF/MEK/ERK signaling. Combining the VEGF and PDGF pathways limits tumor vascularization and endothelial cell proliferation, which retard tumor progression [21]. Sorafenib has been established as the standard of care applying to advanced-stage disease that has demonstrated capability of prolonging survival and delay time of progressing [3]. Single-agent therapy while using Sorafenib reduces the risk

Table 2. Recent advances made in liver transplantation for HCC.

\begin{tabular}{|c|c|c|c|}
\hline & \multicolumn{3}{|c|}{ Recent advances made in liver transplantation for HCC } \\
\hline & Established & Under discussion & Under trial or proposal \\
\hline $\begin{array}{l}\text { Criteria for listing } \\
\text { candidate }\end{array}$ & $\begin{array}{c}\text { The milan criteria : solitary tumor of } \\
\leq 5 \mathrm{~cm} \text { or up to } 3 \text { nodules } \leq 3 \mathrm{~cm} \\
5 \text {-yr survival of } 70 \% \text { with Recurrence } \\
\text { in less than } 10 \%\end{array}$ & $\begin{array}{c}\text { The UCSF criteria: } \\
\text { Single tumor } \leq 6.5 \mathrm{~cm} \text { or } 2-3 \text { tumors } \leq \\
4.5 \mathrm{~cm} \text { with a total tumor diameter } \leq 8 \mathrm{~cm}\end{array}$ & $\begin{array}{l}\text { Add parameters for biologic } \\
\text { features of tumors related to risk } \\
\text { of recurrence } \\
\text { (AFP, PIVKA-II, etc.) }\end{array}$ \\
\hline $\begin{array}{l}\text { Management on the } \\
\text { waiting list (about } 40 \% \\
\text { dropout rate at } 12 \mathrm{mo} \text { ) }\end{array}$ & $\begin{array}{l}\text { Local ablation therapy and TACE are } \\
\text { performed without solid evidence }\end{array}$ & $\begin{array}{l}\text { Different models have been developed } \\
\text { to quantify the risk of death in } \\
\text { neoplastic and non-neoplastic } \\
\text { patients Association with liver resection. } \\
\text { "bridging resection "to transplantation and } \\
\text { "salvage transplantation" } \\
\text { following resection }\end{array}$ & $\begin{array}{l}\text { Application of living donor-LT to } \\
\text { shorten the waiting time } \\
\text { Candidate selection with } \\
\text { information from precedent } \\
\text { therapy (histologic } \\
\text { specimen, response to } \\
\text { locoregional therapy, etc.) }\end{array}$ \\
\hline
\end{tabular}

HCC: Hepatocellular carcinoma; PIVKA: Protein induced by vitamin K absence; AFP: $\alpha$-fetoprotein; LT: Liver transplantation; UCSF: University of California, San Francisco. 
of death by approximately 31\%, while prolonging median survival and progress period by nearly three months [20].

\section{Transarterial Chemoembolization (TACE)}

TACE is considered effective for intermediate-staged patients experiencing unresectable HCC, but not qualifying to undergo ablation therapy and free from invasion or extra hepatic spread [3]. It fits patients with multifocal or large tumour disease that is confined to the liver then the benefits of TACE can be achieved [10]. These are the select conditions in which TACE has been observed to improve the patients' survival [3]. TACE is currently considered the standard of care for those patients undergoing intermediate-stage HCC. Focus of the recent investigations is mainly on establishing whether it is possible to improve the outlook for intermediate-stage disease by combining TACE with Sorafenib [10].

TACE is the most common primary treatment for unresectable HCC and is the recommended first-line treatment fitting the intermediate stage of the disease. Notably, HCC tends to have intense neo-angiogenic activity in its development. The idea behind TACE is that the intra-arterial infusion of a cytotoxic agent and them following it by the embolization of the tumor-feeding blood vessels would cause a strong cytotoxic and ischemic effect. The median survival of untreated patients at the intermediate stage (BCLC B) is $49 \%$ at two years or 16 months. TACE extends this median to 36 - 45 months [5].

In comparison to RFA, patients who have undergone TACE are likely to experience more complications and symptoms. This is because of the fact that, even though the TACE treatment has over the years been widely applied, it is potentially toxic to the liver because of the expected retention and perfusion of chemical medication. Furthermore, the manner the primary HCC nodules are treated by the TACE treatment is sometimes insufficient, necessitating the repetition of TACE procedures. As well, with increment in the number of procedures, more liver deterioration and complication occur [7].

In a study aimed at comparing long-term survival between patient treated with TACE and others who received conservative treatment, TACE exhibits improved survival benefits and higher quality of life for patients having the unresectable HCC in comparison to symptomatic supportive care [22]. This is one of the reasons that render TACE to be considered one of the standard treatment fitting patients with unresectable HCC. This enhanced by the fact that even though Sorafenib has been recommended in treating advanced and unresectable HCC, TACE recommended in treating infiltrating HCC because of the high cost and modest efficacy of Sorafenib treatment [22].

In another study aimed at examining the benefits that are likely to be made in combining Sorafenib with TACE in treating patients with advanced HCC, TACE procedure has a high local tumor control rate while capable of enhancing survival among patients with intermediate HCC. Nevertheless, the hypoxia that TACE causes in the surviving tumor cells causes the release of angiogenic growth factors that cause tumor recurrence and thus poor outcome. This prompts the need to combine it with Sorafenib to prolong the time it takes to progress for those patients with unresectable HCC. This is considering Sorafenib would block the proliferation [23]. In a study, it was advanced that intrahepatic or extracapsular tumor invasions are likely to be experienced after TACE treatment because of incomplete embolization. In their case, they found that TACE is even likely to be counterproductive. This is considering the ischemic injury caused by TACE, which is capable of up-regulating the vascular endothelial growth factor, which is likely to favor HCC growth, metastasis and invasion. It is for this reason that there arose the need of improving the outcome of intermediate HCC patients undergoing TACE treatment [12].

\section{Radiofrequency Ablation (RFA)}

RFA is a local tumor therapy in treating primary and secondary liver tumors. It is conceptualized as a curative therapy option applicable in the very early stage and early stage HCC. In its usage, image guidance facilitates a minimal-invasive application of ablation therapy. In particular, the modality of image guidance critically influences on the efficacy and safety of RFA. The reliance on magnetic resonance imaging in RFA is advantageous considering the advantages it provides in enabling the monitoring of thermal effects, enabling high soft tissue contrast, free selection of imaging planes near real-time fluoroscopic imaging, absence of ionizing radiation and no requirement of iodinated contrast media [24]. RFA is considered in medical oncology to treat hepatic tumors when it would not be feasible to undertake surgery [25]. 
RFA was established in the early 1990s and it is possible for it to be administered during an open surgery, either through laproscopy or percutaneously, even though the latter approach is the most common. In its administration under ultrasound guidance or computed tomography (CT), a needle is inserted into the tumor through the skin. Electrodes that are attached to the tip of the inserted needle deliver the energy that the radiofrequency waves create and the energy induced a thermal injury that is proportionate to the temperature that is achieved and the heating duration. For the tumor tissue to be destroyed, the temperature is maintained at $50^{\circ}$ to $100^{\circ}$ for a duration ranging from 4 to 6 minutes based on the size of the tumor. The destroyed tissues gradually shrink to form a scar. Conventionally, the percutaneous RFA has been applied for tumors that are less than $3 \mathrm{~cm}$ even though its capability of being used for tumors of 3 - $5 \mathrm{~cm}$ and even larger has been described [26].

RFA emerges as the most effective first-line transdermal ablative therapy courtesy of its higher efficacy in relation to being a local cure in comparison to ethanol injection. Ablations the therapy that would be safe and effective in the case of unresectable HCC with its 5-year survival rates comparing well with resection among patients fitting the optimal surgical profile [3]. The survival outcomes of those achieving complete response through RFA can be compared with those that have been treated with hepatic resection [1].

Nevertheless, even after the ablation, frequent recurrences are encountered in the remnant liver because of intrahepatic spread of tumor cells as well as metachronous multicentric carcinogenesis. The rate of recurrence at 5 years is as high as $70 \%-80 \%$. Even though repeated ablation can be undertaken among the patients having recurrent HCC, it is common for tumor to be out of control in the clinical course phases of recurrent recurrence and retreatment. Such explains the poor long-term survival after curative ablation [1].

Following a percutaneous RFA, some of the main complications include skin burns due to the electrodes, hemorrhage, pleural effusion and hepatic abscess. Furthermore, patients who undergo percutaneous RFA are likely to be administered with multiple treatments for them to attain complete necrosis [26]. The need for additional treatment of either percutaneous ethanol injection (PEI) or TACE as follow-up CT scan tends to indicate that the tumor necrosis is incomplete. Without these additional treatments, there is a likelihood of a very high intrahepatic recurrence rate being observed [26].

In evaluating the effective of RFA in patients with intermediate stage HCC on undergoing TACE, RFA emerged as a creative treatment due to its effectiveness and safety in handling early stage small HCC. When compared to TACE treatment, the advantage that RFA lies in its curative local control when treating small HCC. However, the effectiveness of RFA is found to be less favorable in achieving complete tumor necrosis of tumors that are bigger than $5 \mathrm{~cm}$ [12]. This prompts the consideration of combining TACE with RFA, as their combination would be more advantageous than when RFA or TACE are alone. Acting as the downstage treatment, TACE is capable of reducing the tumor burden and thus decrease the viable tumor prior to RFA. This would increase the ablation rates especially for large tumors. Furthermore, repeated TACE procedures are likely to narrow or occlude the main artery that supplies the tumor, resulting to the regeneration of snaking arterioles from the phrenic, gastric, intercostal and the superior mesenteric arteries, which complicate the selective catheterizing of the feeding artery with an aim of controlling residual tumor cells; whereas it is possible for the RFA to abate the refractory tumors directly [12]. Notably, recurrences that arise from curative treatment of HCC during the early post-treatment arise because of the pre-existing microscopic tumor foci that fail to be detected through imaging modalities. Through TACE, it becomes possible to target undetected the satellite lesions that surround the main tumor and label the size and range of the tumor thus providing RFA with guidance. The combination of TACE and RFA is particularly considered effective in locally controlling the medium-sized HCC tumors (3-5 cm) [12].

In an attempt at providing treatment for patients that are not candidates for surgical resection, RFA has emerged as the most widely used ablative technique that has proven to be safe and effective. This has meant that RFA has arose as an adjunctive modality to surgical resection that would apply to colorectal liver metastases in the event that complete resection is not feasible, facilitating improved survival in comparison to nonsurgical treatment. With time, surgical resection has progressively is being combined with RFA in treating patients with bilobar HCC, especially after being deemed to be unresectable if only surgical resection is employed [27].

Image guided RFA is commended for being a minimally invasive therapy option in treating primary and metastatic hepatic malignancies. Particularly in treating HCC, RFA has risen to become an accepted part of the disease therapy concept. Such can be attributed to its associated effective destruction of tumor tissue combined with the preservation of liver tissue and limited negative effect that it has on the liver function that is, in many instances, impaired because of liver cirrhosis. For the benefit to be realized, the modality of image guidance 
ought to ensure that the applicator is precisely targeted to the target tissue and enabling the monitoring of induced tissue coagulation to make sure complete tumor destruction has been achieved and to avoid extensive necrosis of the liver tissue as well as thermal damage of the adjacent structures. It is because of these that the modality of image guidance that has been acknowledged to impact on the efficiency and safety of RFA [24].

Compared to surgical resection, RFA has been found to achieve comparable results in patients whose HCC are less than or equal to $3 \mathrm{~cm}$, but showing inferior outcomes among patients whose HCCs $>3 \mathrm{~cm}$. Apart from tumor size, the effectiveness of RFA is also dependent on tumor location. Hereby, more treatment complications and failures are likely to occur after RFA in the circumstances that HCCs are in high-risk locations that are closely located to the liver capsule, vessels, vital organs or central bile ducts [28]. Notably, high risk locations involve the tumor being situated within $10 \mathrm{~mm}$ of the liver capsules, vital organs (such as gallbladder, colon, stomach, kidneys or common bile duct) the dome of the diaphragm or having direct contact to a vessel that is > $3 \mathrm{~mm}$ in diameter. In the event that the tumor is located near two structures, the nearest one is chosen to be the main structure defining the high-risk location.

\section{Conclusions}

The aggressive nature of HCC is evident and this means that the threat of the disease is anticipated to continue expanding in the coming years. Despite the efforts to develop an optimal treatment, HCC has exhibited a high rate of recurrence. The disease burden is expected to continue increasing particularly considering the rising rates of cirrhosis that tends to linked to obesity, dyslipidemia, type 2 diabetes and hypertension. This means that significant focus needs to shift to developing means of achieving efficient and effective care.

Management of HCC will continue being dependent on tumor number, tumor size, tumor stage, patient performance status and the compromised liver function, which are based on the BCLC staging system [29]. Patients whose disease is in its early phases would fit the potentially curative treatments therapies such as liver resection, local ablation and liver transplantation, with the possibility of achieving 5 -year survival rates of $60-70 \%$ among some sell-selected patients [10]. However, there are patients that are not suitable candidates for the curative surgical resection methods due to the manner the tumors are distributed, poor liver function or presence extra hepatic disease, which make the percutaneous radiofrequency ablation to be the preferred treatment [26].

Significant advances have been achieved to manage the condition and TACE and RFA stand out among them. Particularly in early stages, RFA has proved equal to surgical resection if the patients are well selected. Whereas, even though hampered by complications, the efficacy and safety of TACE has been improving for intermediate HCC. For advanced HCC, chemotherapeutic agents such as Sorafenib are considered. It is expected that with HCC rising more advanced treatments and therapies will continue emerging (Table 3 [30]).

Notably, the non-surgical therapies are evidently considered to extend the patient's survival to possibly downstage the tumor to facilitate liver transplantation and surgical resection. However, considering the lack of effective chemotherapy in treating HCC, complete tumor removal provides the only available option of achieving long-term cure. Nevertheless, surgical operations involving liver transplantation and surgical resection would apply to patients with early stage cancer that is confined to the liver. At the same time, it emerges that even when confined to the liver, it is not outright that every patient will be a candidate for surgery and may call for the consideration of non-surgical treatments approaches.

An evident aspect, considering the link that HCC has with various other ailments, managing it would only be

Table 3. Treatment guidance based on BCLC staging system for HCC.

\begin{tabular}{|c|c|c|c|c|}
\hline \multicolumn{5}{|c|}{ Treatment guidance based on BCLC staging system for HCC } \\
\hline BCLC stage & Tumour stage & $\mathrm{CP}$ class & ECOG Ps & Recommended \\
\hline Very early (0) & $1 \mathrm{HCC}<2 \mathrm{~cm}$ & A & 0 & $\begin{array}{l}\text { Resection } \\
\text { transplantation }\end{array}$ \\
\hline Early (A) & $1 \mathrm{HCC}<5 \mathrm{~cm}$ or 3 nodules $<3 \mathrm{~cm}$ & A or B & 0 & RFA \\
\hline Intermediate(B) & Multinodular & A or B & 0 & TACE \\
\hline Advanced (C) & Portal invasion, N1M1 & A or B & $1-2$ & Sorafenib \\
\hline End-stage (D) & Any & $\mathrm{C}$ & $>2$ & Symptomatic treatment \\
\hline
\end{tabular}


effectively achieved if a multidisciplinary setting were considered. Apart from hepatologists, professionals from other medical disciplines such as oncologists, hepatobiliary and transplant surgeons, palliative care specialists and interventional radiologists need to be considered to facilitate comprehensive HCC treatment and management.

\section{References}

[1] Asaoka, Y., Tateishi, R., Nakagomi, R., et al. (2014) Frequency of and Predictive Factors for Vascular Invasion after Radiofrequency Ablation for Hepatocellular Carcinoma. PLoS One, 9, e111662.

[2] Cucchetti, A., Piscaglia, F., Cescon, M., et al. (2013) An Explorative Data-Analysis to Support the Choice between Hepatic Resection and Radiofrequency Ablation in the Treatment of Hepatocellular Carcinoma. Digestive and Liver Disease, 46, 257-263. http://dx.doi.org/10.1016/j.dld.2013.10.015

[3] Shaya, F.T., Breunig, I.M., Seal, B., Mullins, C.D., Chirikov, V.V. and Hanna, N. (2014) Comparative and Cost Effectiveness of Treatment Modalities for Hepatocellular Carcinoma in SEER-Medicare. PharmacoEconomics, 32, 63-74. http://dx.doi.org/10.1007/s40273-013-0109-7

[4] Lee, D.H., Lee, J.M., Lee, J.Y., et al. (2014) Non-Hypervascular Hepatobiliary Phase Hypointense Nodules on Gadoxetic Acid Enhanced MRI: Risk of HCC Recurrence after Radiofrequency Ablation. Journal of Hepatology, 14, In Press. http://dx.doi.org/10.1016/j.jhep.2014.12.015

[5] Fatourou, E.M. and Tsochatzis, E.A. (2014) ART and Science in Using Transarterial Chemoembolization for Retreating Patients with Hepatocellular Carcinoma. Hepatobiliary Surgery and Nutrition, 3, 415-418.

[6] Jelic, S. and Sotiropoulos, G.C. (2010) Hepatocellular Carcinoma: ESMO Clinical Practice Guidelines for Diagnosis, Treatment and Follow-Up. Annals of Oncology, 21, v59-v64. http://dx.doi.org/10.1093/annonc/mdq166

[7] Wang, Y.B., Chen, M.H., Yan, K., Yang, W., Dai, Y. and Yin, S.S. (2007) Quality of Life after Radiofrequency Ablation Combined with transcatheter Arterial Chemoembolization for Hepatocellular Carcinoma: Comparison with Transcatheter Arterial Chemoembolization Alone. Quality of Life Research, 16, 389-397. http://dx.doi.org/10.1007/s11136-006-9133-9

[8] Zhu, S.L., Ke, Y., Peng, Y.C., et al. (2014) Comparison of Long-Term Survival of Patients with Solitary Large Hepatocellular Carcinoma of BCLC Stage A after Liver Resection or Transarterial Chemoembolization: A Propensity Score Analysis. PLoS ONE, 9, e115834. http://dx.doi.org/10.1371/journal.pone.0115834

[9] EASL (2012) EASL-EORTC Clinical Practice Guidelines: Management of Hepatocellular Carcinoma. Journal of Hepatology, 56, 908-943.

[10] Ma, Y.T. and Palmer, D.H. (2012) Impact of Restricting Access to High-Cost Medications for Hepatocellular Carcinoma. Expert Review of Pharmacoeconomics \& Outcomes Research, 12, 465-473. http://dx.doi.org/10.1586/erp.12.33

[11] Topp, Z.Z. and Sigal, D.S. (2013) Beyond Chemotherapy: Systemic Treatment Options for Hepatocellular Carcinoma. Translational Cancer Research, 2, 482-491.

[12] Yin, X., Zhang, L., Wang, Y.H., Zhang, B.H., Gan, Y.H., Ge, N.L., et al. (2014) Transcatheter Arterial Chemoembolization Combined with Radiofrequency Ablation Delays Tumor Progression and Prolongs Overall Survival in Patients with Intermediate (BCLC B) Hepatocellular Carcinoma. BMC Cancer, 14, 849.

[13] Liang, J.D., Ping, X.O., Tseng, Y.J., Huang, G.T., Lai, F. and Yang, P.M. (2014) Recurrence Predictive Models for Patients with Hepatocellular Carcinoma after Radiofrequency Ablation Using Support Vector Machines with Feature Selection Methods. Computer Methods and Programs in Biomedicine, 117, 425-434. http://dx.doi.org/10.1016/j.cmpb.2014.09.001

[14] Lee, S., Yoon, S.H., Park, J.Y., Kim do, Y., Ahn, S.H., Han, K.H. and Choi, H.J. (2012) Sorafenib versus Cytotoxic Chemotherapy for Patients with Advanced Hepatocellular Carcinoma: A Retrospective, Single-Institution Study. Investigational New Drugs, 30, 1150-1157. http://dx.doi.org/10.1007/s10637-011-9634-4

[15] Chen, K., Chen, G., Wang, H., Li, H., Xiao, J., Duan, X., et al. (2014) Increased Survival in Hepatocellular Carcinoma with Iodine-125 Implantation plus Radiofrequency Ablation: A Prospective Randomized Controlled Trial. Journal of Hepatology, 61, 1304-1311. http://dx.doi.org/10.1016/j.jhep.2014.07.026

[16] Kishi, Y., Hasegawa, K., Sugawara, Y. and Kokudo, N. (2011) Hepatocellular Carcinoma: Current Management and Future Development-Improved Outcomes with Surgical Resection. International Journal of Hepatology, 2011, Article ID: 728103. http://dx.doi.org/10.4061/2011/728103

[17] Zhong, J.H., Li, H., Xiao, N., Ye, X.-P., Ke, Y., Wang, Y.-Y., et al. (2014) Hepatic Resection Is Safe and Effective for Patients with Hepatocellular Carcinoma and Portal Hypertension. PLOS ONE, 9, e108755. http://dx.doi.org/10.1371/journal.pone.0108755

[18] Zhang, Q., Chen, H., Li, Q., Zang, Y.J., Chen, X.G., Zou, W.L., et al. (2011) Combination Adjuvant Chemotherapy 
with Oxaliplatin, 5-Fluorouracil and Leucovorin after Liver Transplantation for Hepatocellular Carcinoma: A Preliminary Open-Label Study. Investigational New Drugs, 29, 1360-1369. http://dx.doi.org/10.1007/s10637-011-9726-1

[19] Morise, Z., Kawabe, N., Tomishige, H., Nagata, H., Kawase, J., Arakawa, S., et al. (2014) Recent Advances in the Surgical Treatment of Hepatocellular Carcinoma. World Journal Gastroenterology, 20, 14381-14392. http://dx.doi.org/10.3748/wjg.v20.i39.14381

[20] Cao, H., Phan, H. and Yang, L.X. (2012) Improved Chemotherapy for Hepatocellular Carcinoma. Anticancer Research, 32, 1379-1386.

[21] Ahn, H.K., Lee, S., Sun, J.M., Lee, J., Park, S.H., Park, J.O., et al. (2012) Sequential Therapy with Sunitinib and Sorafenib in Metastatic Hepatocellular Carcinoma. Investigational New Drugs, 30, 1768-1772. http://dx.doi.org/10.1007/s10637-011-9707-4

[22] Dai, Q.S., Gu, H.L., Ye, S., Zhang, Y.J., Lin, X.J., Lau, W.Y., et al. (2014) Transarterial Chemoembolization vs. Conservative Treatment for Unresectable Infiltrating Hepatocellular Carcinoma: A Retrospective Comparative Study. Molecular and Clinical Oncology, 2, 1047-1054.

[23] Shao, W., Zhang, F., Cong, N., Li, J. and Song, J. (2014) Transarterial Chemoembolization Combined with Sorafenib for Advanced Hepatocellular Carcinoma. Oncology Letters, 8, 2263-2266.

[24] Clasen, S., Rempp, H., Hoffmann, R., Graf, H., Pereira, P.L. and Claussen, C.D. (2013) Image-Guided Radiofrequency Ablation of Hepatocellular Carcinoma (HCC): Is MR Guidance More Effective than CT Guidance? European Journal of Radiology, 83, 111-116. http://dx.doi.org/10.1016/j.ejrad.2013.09.018

[25] Caminiti, I.M.V., Ferraioli, F., Formisano, A. and Martone, R. (2012) Three Dimensional Optimal Current Patterns for Radiofrequency Ablation Treatments. COMPEL, 31, 985-995. http://dx.doi.org/10.1108/03321641211209852

[26] Xie, X., Dendukuri, N. and McGregor, M. (2010) Percutaneous Radiofrequency Ablation for the Treatment of Early Stage Hepatocellular Carcinoma: A Health Technology Assessment. International Journal of Technology Assessment in Health Care, 26, 390-397. http://dx.doi.org/10.1017/S0266462310001029

[27] Zhang, T., Zeng, Y., Huang, J., Liao, M. and Wu, H. (2014) Combined Resection with Radiofrequency Ablation for Bilobar Hepatocellular Carcinoma: A Single-Center Experience. Journal of Surgical Research, 191, 370-378. http://dx.doi.org/10.1016/j.jss.2014.03.048

[28] Lin, J.W., Lin, C.C., Chen, W.T. and Lin, S.M. (2014) Combining Radiofrequency Ablation and Ethanol Injection May Achieve Comparable Long-Term Outcomes in Larger Hepatocellular Carcinoma (3.1-4 cm) and in High-Risk Locations. The Kaohsiung Journal of Medical Sciences, 30, 396-401. http://dx.doi.org/10.1016/j.kjms.2014.04.006

[29] Raza, A. and Sood, G.K. (2014) Hepatocellular Carcinoma Review: Current Treatment, and Evidence-Based Medicine. World Journal Gastroenterology, 20, 4115-4127. http://dx.doi.org/10.3748/wjg.v20.i15.4115

[30] Sherman, M., Burak, K., Maroun, J., Metrakos, P., Knox, J.J., Myers, R.P., et al. (2011) Multidisciplinary Canadian Consensus Recommendations for the Management and Treatment of Hepatocellular Carcinoma. Current Oncology, 18, 228-240. http://dx.doi.org/10.3747/co.v18i5.952 